\title{
CLINICAL RESEARCH ARTICLE OPEN
}

Assessment of neonatal EEG background and neurodevelopment in full-term small for their gestational age infants

\author{
José R. Castro Conde ${ }^{1,2}$, Candelaria González Campo ${ }^{2}$, Nieves L. González González ${ }^{1,3}$, Beatriz Reyes Millán ${ }^{4}$, Desiré González Barrios ${ }^{5}$, \\ Alejandro Jiménez Sosa ${ }^{6}$ and Itziar Quintero Fuentes ${ }^{7}$
}

BACKGROUND: Delayed brain function development in small-gestational-age (SGA) infants has been reported. We aimed to quantify rates of immature neonatal EEG patterns and their association with neurodevelopment in SGA full-term neonates. METHODS: Using a cohort design, 50 SGA (birthweight <10th percentile) and 44 appropriate-gestational-age (AGA) term neonates underwent continuous video-EEG recordings lasting $>3 \mathrm{~h}$. Seventy-three of them were assessed at 2-years-old using Bayley-IIIScales. For EEG analysis, several segments of discontinuous/alternating EEG tracings were selected. Main outcomes measured: (1) Visual analysis (patterns of EEG maturity); (2) Power spectrum in $\delta, \theta, a$ and $\beta$ frequency bands; and (3) scores in motor, cognitive and language development.

RESULTS: (1) SGA infants, compared to AGA, showed: (a) higher percentages of discontinuous EEG, both asynchrony and interhemispheric asymmetry, and bursts with delta-brushes, longer interburst-interval duration and more transients/hour; (b) lower relative power spectrum in $\delta$ and higher in $\alpha$; and (c) lower scores on motor, language and cognitive neurodevelopment. (2) Asymmetry $>5 \%$, interburst-interval $>5 \mathrm{~s}$, discontinuity $>11 \%$, and bursts with delta-brushes $>11 \%$ were associated with lower scores on Bayley-III.

CONCLUSIONS: In this prospective study, SGA full-term neonates showed high rates of immature EEG patterns. Low-birthweight and immaturity EEG were both correlated with low development scores.

Pediatric Research (2020) 88:91-99; https://doi.org/10.1038/s41390-019-0693-0

\section{INTRODUCTION}

Small for their gestational age (SGA) infants exhibit deficits in their neurodevelopmental outcomes at 6 years of age. ${ }^{1}$ Disturbances in metabolism, microstructure, hemodynamics, morphology and brain connectivity have been found in late-onset intrauterine growth restriction (IUGR) children., ${ }^{2,3}$

At the acute moment of a cerebral injury, brain activity shows several degrees of electrophysiological depression. However, intrauterine unfavorable conditions can cause a slight but persistent brain insult that eventually causes irreversible chronic brain damage. ${ }^{4}$ In this "chronic state", the neurophysiological abnormalities are replaced by dysmaturity and/or disorganization. ${ }^{5}$

The brain undergoes dramatic structural maturation during the last months of intrauterine life, allowing experienced clinicians to estimate the gestational age (GA) with differences of up to a week. ${ }^{6}$ These maturational EEG changes continue in the postnatal life and are especially pronounced in the first 3 days of life as a result of adaptation to postnatal life. ${ }^{7,8}$ The most recent studies in the development of brain activity have relied on quantitative analyses of EEG activity. ${ }^{9-11}$

Spectral EEG analysis and neonatal aEEG findings have found delayed maturation and less optimal scores on early EEG in preterm ${ }^{12,13}$ and full-term SGA neonates. ${ }^{14}$ But, specific patterns of maturity on the visual background EEG analysis regarding neonatal brain function development and its prognostic implications in IUGR children have never been analyzed.

Our hypothesis was that SGA term infants have high rates of immature patterns on the EEG background that could be associated to development deficits. Using the visual and spectral analysis, the objectives of the present study were: (1) to quantify rates of well-known patterns of EEG maturity in low-birth-weight term babies at $48-72 \mathrm{~h}$ after birth, and (2) to use the measurements obtained in these EEG variables as prognostic markers on the neurodevelopmental assessment in early childhood.

\section{METHODS}

Participants and design

Using a cohort design, between March 2014 and January 2016, 51 term SGA and 67 term appropriate-gestational-age (AGA) infants matched by GA were recruited. SGA babies were selected from IUGR fetuses diagnosed in the middle of the 2 nd to the 3rd trimester by Doppler (middle cerebral artery and umbilical artery) and biometric measurements (abdominal and head circumference, and femur

\footnotetext{
${ }^{1}$ Department of Obstetrics and Gynecology, and Pediatrics, Universidad de La Laguna, La Laguna, Spain; ${ }^{2}$ Department of Neonatology, Hospital Universitario de Canarias, La Laguna, Spain; ${ }^{3}$ Department of Obstetrics and Gynecology, Hospital Universitario de Canarias, La Laguna, Spain; ${ }^{4}$ Department of Neonatology, Hospital Universitario Nuestra

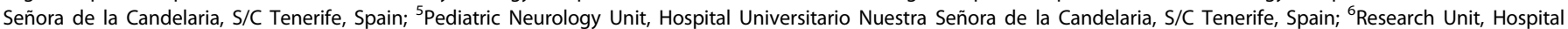
Universitario de Canarias. Ofra s/n, 38320 La Laguna, Spain and ${ }^{7}$ Department of Clinical Psychology, Universidad de La Laguna, La Laguna, Spain Correspondence: José R. Castro Conde (jcastro@ull.edu.es)
} 
length) of the fetal ultrasound, and confirmed by presenting a birthweight $<10$ th percentile according to tables of intrauterine growth customized in our population. ${ }^{15} \mathrm{GA}$ was calculated from the first day of the mother's last menstrual period and confirmed by prenatal ultrasound in the first trimester.

All SGA children of the present study were diagnosed prenatally and their etiology was evaluated following the guidelines of the Sociedad Española de Ginecología y Obstetricia (SEGO). ${ }^{16}$ The postnatal etiological evaluation was conditioned by the clinical requirements of each baby. Inclusion criteria were $\mathrm{GA}>37$ weeks, no neonatal resuscitation, 5-minute Apgar score $>7$ and umbilical cord $\mathrm{pH}>7.10$. Exclusion criteria were maternal body mass index $<20$ or $>30$, chronic maternal disease (diabetes, epilepsy, moderate/severe asthma), obstetric pathology (hypertension, metrorrhagia), noxious habits (alcohol, drug use), multiple pregnancy, major congenital malformations or recognized/suspected genetic syndrome, congenital infections, birthweight $>4 \mathrm{~kg}$ and admission to the neonatal special care unit.

All neonates underwent Amiel-Tison neurological assessment ${ }^{17}$ at the third day of life. Only children with an optimal score were included.

Standard protocol approvals, registrations and patient consent. See in Statement of Ethics.

\section{EEG procedure}

All infants underwent continuous video-EEG recordings at $48-72 \mathrm{~h}$ after birth in the nursery within a single postnatal ward. The duration of each EEG recording was $>3 \mathrm{~h}$. Several montages were used with electrodes placed according to the international 10-20 system adapted for neonates. The control of environmental variables, the polygraph and the technical adjustments of EEG recording complied with previous recommendations. ${ }^{18}$

Visual analysis

The visual analysis included at least $1 \mathrm{~h}$ of sleep recording. Sleep states regarding behavioral and EEG parameters were classified as quiet sleep (QS), active sleep (AS) or indeterminate sleep (IS), in accordance with previous recommendations. ${ }^{19}$ We calculated the percentages of time of both discontinuous pattern and tracé alternant in relation to the sum of IS and QS of the entire EEG recording. We defined discontinuous pattern as bursts of EEG activity with amplitude $>50 \mu \mathrm{V}$ separated by low-voltage activity $<30 \mu \mathrm{V}$ (if $\geq 30 \mu \mathrm{V}=$ alternating tracing) for $>1 \mathrm{~s}$. The following specific EEG patterns of GA maturity were analyzed exclusively during discontinuity or alternating tracing: (1) maximum duration in seconds of interburst interval (IBI), (2) percentage of bursts with delta brushes, (3) percentage of bursts with both interhemispheric asynchrony and asymmetry, and (4) transients/hours (Fig. 1).

The definitions of these variables were strictly adjusted to those used previously to analyze the EEG in healthy term neonates: ${ }^{8}$

Interburst intervals of inactivity or hypoactivity (IBI). Epochs with an EEG activity $<30 \mu \mathrm{V}$ on all channels for $>1 \mathrm{~s}$.

Delta brushes. Delta waves of $500-1500 \mathrm{~ms}$ duration with amplitude of $50-250 \mu \mathrm{V}$, with rapid activity $(8-20 \mathrm{~Hz})$ of amplitude $>15 \mu \mathrm{V}$ superimposed on the ascending slope of the first slow wave of the burst.

Inter-hemispheric asynchrony. Burst onset between hemispheres separated by $\geq 1.5 \mathrm{~s}$.

Inter-hemispheric asymmetry. Bursts with a difference of amplitude $\geq 50 \%$ between the two hemispheres and/or with superimposed rapid activity in only one hemisphere.

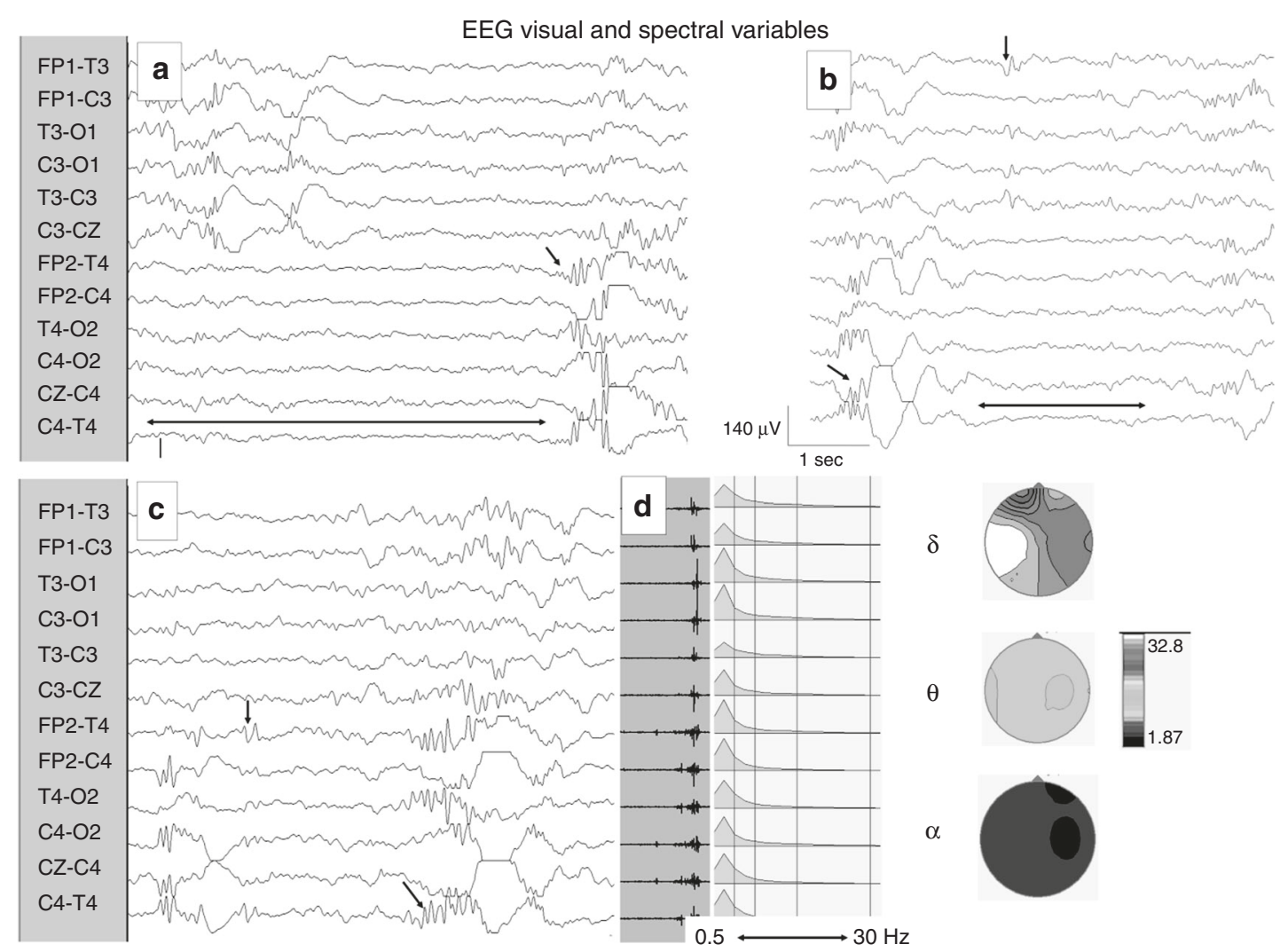

Fig. 1 Examples the EEG variables studied in the visual and spectral analysis in an SGA term infant. $\mathbf{a}$ and $\mathbf{b}$ Discontinuous EEG tracings showing interhemispheric asynchrony and asymmetry, respectively, with transient rolandic sharp waves (vertical arrow), interburst interval (two-headed arrow) and delta brushes in the bursts (Inclined arrows). c Interhemispheric asymmetry of the bursts on the EEG background with trace alternant and transient temporal sharp waves (vertical arrow). d Graphs and topographic maps of absolute power spectrum in the $\delta, \theta$ and $a$ frequency bands; the values in the color scale represent absolute power spectrum in $\mu \mathrm{V}^{2}$. 
Table 1. Results obtained from the EEG parameters evaluated in the visual analysis, expressed as mean, standard deviation, statistical significance, and cut-off points of maximum likelihood to discriminate both study groups.

\begin{tabular}{|c|c|c|c|c|c|}
\hline EEG variables & Interrater agreement ICC $(95 \% \mathrm{CI})^{\mathrm{b}}$ & \multicolumn{2}{|c|}{ Group of study } & Sig. & Cut-off points \\
\hline$\%$ Tracé alternant & $0.93(0.90-0.96)$ & $26.37 \pm 20.72$ & $51.94 \pm 21.18$ & $<0.001$ & $<30 \%$ \\
\hline \% Discontinuous tracing & $0.97(0.96-0.98)$ & $37.62 \pm 26.79$ & $5.53 \pm 13.032$ & $<0.001$ & $>11 \%$ \\
\hline \% Bursts with Delta Brushes & $0.83(0.68-0.92)$ & $23.78 \pm 18.04$ & $6.45 \pm 6.13$ & $<0.001$ & $>11 \%$ \\
\hline Maximum IBI duration (s) & $0.77(0.66-0.85)$ & $6.84 \pm 2.60$ & $2.82 \pm 2.67$ & $<0.001$ & $>5 \mathrm{~s}$ \\
\hline No. transients/hour & $0.73(0.61-0.89)$ & $17.68 \pm 13.02$ & $8.20 \pm 6.48$ & $<0.001$ & $>11$ \\
\hline
\end{tabular}

Transients. Positive or negative waves with amplitude $>50 \mu \mathrm{V}$ and duration between 100 and 400 ms clearly present only in the interburst epochs.

Examples of some of these EEG variables are shown in Fig. 1a-c. Each EEG parameter of the visual analysis, including artifact rejection, was measured individually and independently by two neuropediatricians experienced in neonatal brain monitoring who were unaware of the baby's birth weight. We accepted an EEG feature only when both observers agreed. Since all variables of the visual EEG analysis were continuous, the interrater reliability analysis was performed using intraclass correlation coefficient. ${ }^{20}$ The interrater agreement rate (mean and $95 \% \mathrm{Cl}$ ) for all these variables is shown in Table 1.

\section{Spectral analysis}

In the valid EEG recording of each neonate, both neuropediatricians independently selected all segments of the total EEG recording exclusively with tracé alternant and/or discontinuous pattern regardless of the sleep state and without any evident artifacts. All EEG segments with full concordance between the two observers, lasting $\geq 1 \mathrm{~min}$ each (time considered to define a behavior state), were included in the analysis. The cumulative recording time of the selected EEG segments ranged between 15 and $21 \mathrm{~min}$. The analysis was performed using Persyst Insight II EEG analysis software (version 6.0.0/Build 632-XLDB; Persyst Development Corporation, Prescott, AZ). The software settings complied with previous studies. 8,21

Spectral analysis included relative power spectrum in the frequency bands: $\delta(0.5-4 \mathrm{~Hz}) ; \theta(4-8 \mathrm{~Hz}) ; a(8-13 \mathrm{~Hz}) ;$ and $\beta$ $(13-30 \mathrm{~Hz})$. Relative power spectrum was defined as the absolute power spectrum for each frequency band divided by the sum of absolute power spectrum of all frequencies, expressed as a percentage. The EEG channels used for the measurements of the power spectrum were: Fp1-T3, Fp1-C3, C3-Cz C3-T3; O1-C3, O1-T3, $\mathrm{Fp} 2-\mathrm{T} 4$, Fp2-C4, C4-Cz C4-T4; O2-C4, and O2-T4 (Fig. 1d). We computed the total relative power spectrum (averaging all EEG channels) in each frequency band as well as the $\delta / \theta, \delta / a$ and $\delta / \beta$ ratios.

Neurodevelopment

A total of 73 (41 SGA and 32 AGA) 2-year-old (range 24-26 months) children were examined (Flow chart in Fig. 2). Using the Bayley Scales of Infant Development Third Edition (Bayley-III), neurodevelopmental data were collected and analyzed by two psychometricians who were unaware of the children's birthweight.

Statistical analysis

We carried out a pre-hoc exploratory analysis with 30 children in each group and we observed that the mean difference in the discontinuity percentage was $18.74 \%(24.55 \% \pm 21.24 \%$ vs $5.10 \%$ $\pm 8.27 \%$ in SGA and AGA, respectively). For a confidence level of $95 \%$ and a power of more than $99 \%$, at least 40 children per group are required to demonstrate that the percentages of discontinuous patterns are higher in the SGA group.

ROC curves analyses, including Youden index, were used to obtain the cut-off point of each visual EEG variable with the maximum likelihood to discriminate between SGA and AGA neonates. Regardless of birthweight, we used these cut-off points as neonatal specific markers of maturity EEG, establishing two groups: (1) children with higher values, and (2) children with values equal to or lower than the cut-off point. Those whose EEG backgrounds showed $\leq 2$ variables with values above the cut-off point were classified as mature and those with $>3$ as immature.

For comparisons between birthweight (SGA vs AGA), sex (males vs females), EEG maturity (mature vs immature), and children in the follow-up (lost vs non-lost) groups for EEG visual and spectral variables, we used Mann-Whitney $U$ test. The correlations between the continuous variables of the visual analysis with those of the power spectrum and between the variables of the spectral analysis with the development scores were estimated using the Spearman correlation coefficient (two-sided) and using the Bonferroni correction to correct multiple comparisons.

Using multivariate linear regression, we tested the independent effects of the birthweight group on EEG variables and development scores, controlling the confounding effects of perinatal factors (maternal smoking, sex and cesarean delivery).

Otherwise all $P$ values $<0.05$ were considered statistically significant. Statistical analysis were performed with SPSS v. 21.0 (Chicago, IL), Statistica v. 5.0 (StatSoft, OK) and MedCalc Statistical Software version 13.0.2 (MedCalc Software bvba, Ostend, Belgium; http://www.medcalc.org).

\section{RESULTS}

Perinatal data

The main perinatal data are shown in Table 2. There were 38 SGA infants who had birthweight $<3$ rd percentile and the remaining 
Full-term neonates included and children assessed at two-year-old

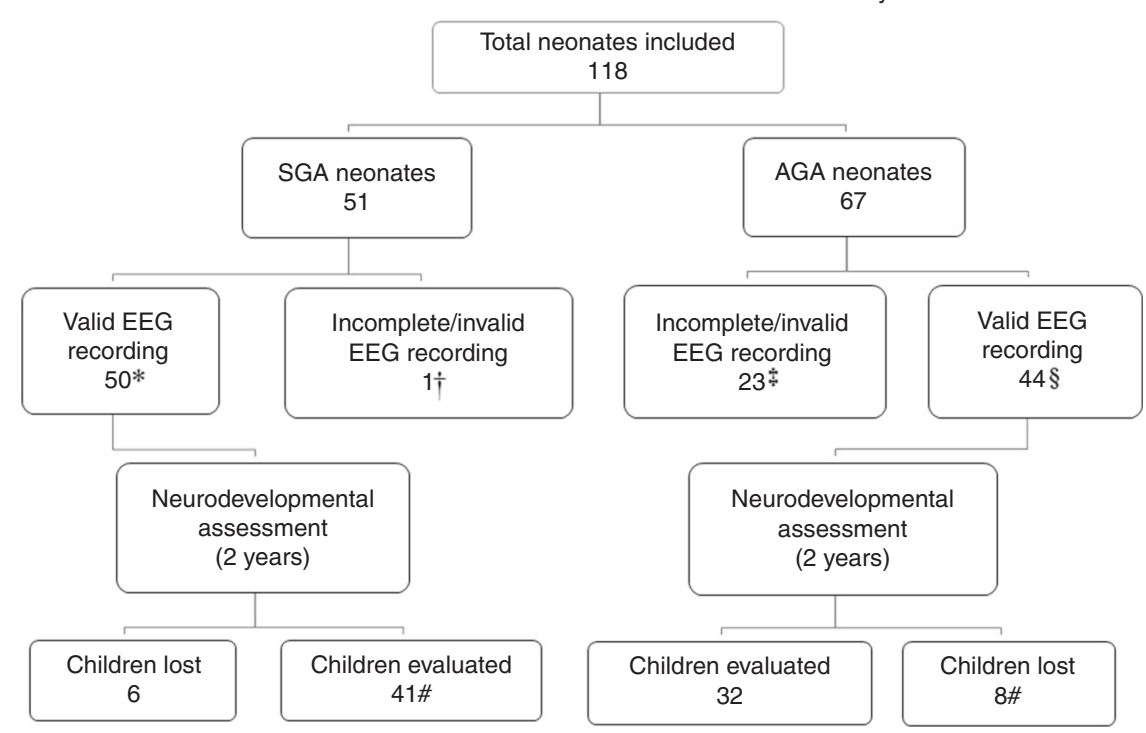

Fig. 2 Flow chart of children included in the study sample. *It was necessary to practice more than one EEG recording of different days in 17 newborns. † His parents refused a new EEG recording. ₹ In none of them could a new EEG recording be repeated. § The EEG recording was repeated in four of them. \# There were three SGA children and four other AGA children who did not collaborate.

\begin{tabular}{|c|c|c|c|}
\hline Perinatal data & $\mathrm{SGA}(n=50)$ & AGA $(n=44)$ & $\begin{array}{l}\text { Significance } \\
P \text { value }\end{array}$ \\
\hline Birthweight (g) & $2140.14 \pm 330.09$ & $2987.16 \pm 522.32$ & $<0.001$ \\
\hline Body length $(\mathrm{cm})$ & $44.11 \pm 3.23$ & $49.47 \pm 2.40$ & 0.009 \\
\hline Male sex & $27 / 50(54 \%)$ & $21 / 44(47.7 \%)$ & 0.44 \\
\hline Head circumference & $32.20 \pm 1.50$ & $34.06 \pm 1.59$ & $<0.001$ \\
\hline Birthweight percentile & $1.87 \pm 2.05$ & $47.98 \pm 27.45$ & $<0.001$ \\
\hline Gestational age & $37.73 \pm 1.73$ & $38.10 \pm 2.00$ & 0.69 \\
\hline Maternal age & $33.14 \pm 5.22$ & $33.75 \pm 5.20$ & 0.58 \\
\hline Maternal smoking & (11/50) $22 \%$ & (3/44) $6.8 \%$ & 0.046 \\
\hline Cesarean delivery & (18/50) $36 \%$ & (8/44) $18.2 \%$ & 0.07 \\
\hline Apgar (1 min) & $8.44 \pm 1.36$ & $8.59 \pm 1.07$ & 0.56 \\
\hline Apgar (5 min) & $8.89 \pm .451$ & $8.92 \pm 0.363$ & 0.79 \\
\hline Umbilical cord PH & $7.239 \pm .069$ & $7.256 \pm 0.068$ & 0.34 \\
\hline Cerebroplacental ratio ${ }^{\mathrm{a}}$ & $1.215 \pm .557$ & $1.694 \pm 1.281$ & 0.08 \\
\hline \multicolumn{4}{|l|}{ Maternal educational level } \\
\hline Primary school & (7/50) $14 \%$ & (5/44) $11.4 \%$ & 0.77 \\
\hline High school & (22/50) $44 \%$ & (26/44) 59.1\% & 0.23 \\
\hline $\begin{array}{l}\text { College/university } \\
\text { education }\end{array}$ & (21/50) $42 \%$ & (13/44) $39.5 \%$ & 0.60 \\
\hline
\end{tabular}

12 babies $\mathrm{SGA} \geq 3$ rd and $<10$ th percentile. Fetal brain and umbilical Doppler ultrasound was performed in all SGA infants. The cerebral/placenta ratio (CPR) calculated by the pulsatility index, was (1) <3rd percentile in $15 \mathrm{SGA}$; and (2) between $\geq 3 \mathrm{rd}$ and $<10$ th percentile in another 13 SGA fetuses. No emergent cesarean delivery, delivery room resuscitation or respiratory support was necessary in any infant.

The invalid or incomplete EEG recordings were due to the prolonged duration of the monitoring video-EEG without obtaining one full sleep-wake cycle and/or discomfort of the baby. It was possible to practice $\geq$ one new EEG recording until getting a valid one in 17 SGA and four AGA infants. Parents refused another new
video-EEG recording in one SGA, and in 23 AGA. A valid EEG recording was obtained in 50 SGA and in 44 AGA neonates, who were the babies included for the study.

\section{Visual EEG analysis}

EEG immaturity was found in 39 SGA and only in four AGA neonates $(P<0.001)$. Table 1 shows the results obtained in all different variables of background EEG visual analysis, including cut-off points obtained from of the ROC curve analysis with a positive predictive value $>80 \%$ and $P$ value $<0.001$ belonging to the group of SGA infants. The main results in visual EEG analysis comparing both birthweight groups are shown in Fig. 3a. No epileptiform EEG discharges were recorded in any neonate. No significant differences were found between sex (males vs females) and children in the follow-up (lost vs non-lost) groups for visual and spectral EEG variables. No contribution of confounding factors (cesarean delivery and smoking) were found on any EEG variable.

No significant differences were found in any EEG variable between SGA babies with birthweight $<3$ rd percentile and those with birthewight $\geq 3 \mathrm{rd}$ and $<10$ th percentile. The results obtained when comparing three birthweight groups (SGA $<3$ rd percentile, $S G A \geq 3$ rd and $<10$ th percentile, and AGA babies) are graphically shown in Supplemental Fig. S1 (online).

Quantitative analysis

Compared with the AGA, the neonates SGA group showed lower total relative power spectrum in $\delta$ and higher faster frequency bands. (Fig. 3b). The results of the total relative power spectrum for each frequency band and the ratios, expressed as mean, 95\% confidence interval and statistical significance, are shown in Supplemental Table S1 (online).

After Bonferroni correction, we found a significant correlation between relative power spectrum in: (1) $\delta, a$ and $\beta$ frequency bands with percentage of discontinuous pattern $(r=-0.39$, $P<0.001 ; r=0.41, P<0.001 ; r=0.44, P<0.001$, respectively), (2) $a$ with percentage of bursts with delta brushes $(r=0.30, P=$ $0.004)$, and (3) $\beta$ with percentage of asymmetry $(r=0.29, P=$ 0.007 ). No other statistically significant correlations were found between visual and spectral EEG variables. 
a
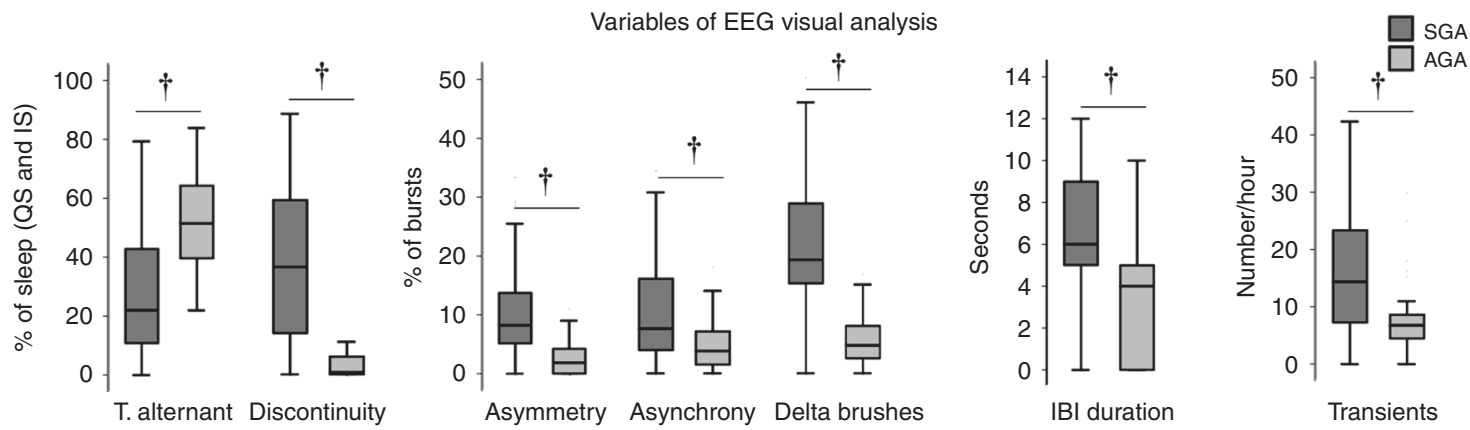

b

Total relative power spectrum in each frequency band and $\delta / \theta, \delta / \alpha$ and $\delta / \beta$ ratios
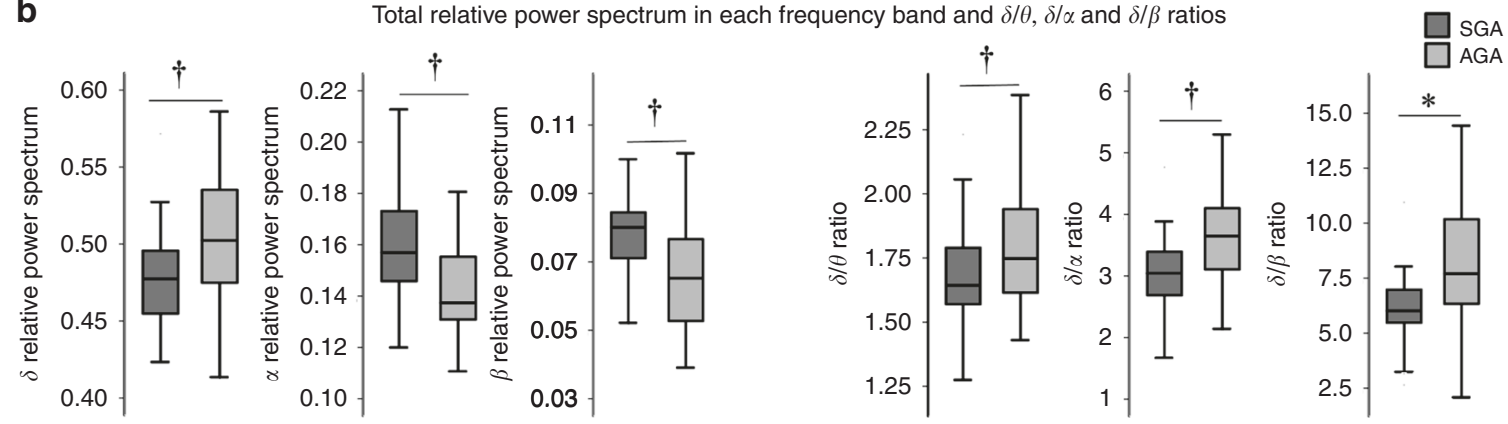

Fig. 3 Main results of visual and spectral analysis. Box plots represent medians, interquartile ranges (P25 to P75), and min-max (whiskers) of the main results of the visual EEG analysis (a) and of the relative power spectrum (b). ${ }^{*} P<0.05 ;+P<0.01$.

Neurodevelopment

Low birthweight and high rates of some immature EEG patterns both correlated with each other and, independent of other variables, were associated with significantly lower scores in one or more development domains. Table 3 shows these findings in detail. Taking into account only the group of SGA babies, we found: (1) no differences in the development scores between the mature and immature EEG, (2) using cut-off point, only interhemispheric asymmetry $>5 \%$ presented significantly lower scores in the language domain $(95.76 \pm 12.03)$ compared to SGA with asymmetry $\leq 5 \%(104.55 \pm 13.71)(P=0.049)$, and (3) using Spearman correlation coefficient, a significant negative correlation between asymmetry and motor development $(r=-0.310 ; P=$ 0.02 ) and borderline not significant in the language domain $(r=-0.236, P=0.061)$. When considering exclusively the AGA group, we also found a significant negative correlation between interhemispheric asymmetry and motor development $(r=-0.328$; $P=0.031$ ).

In the quantitative analysis, we found: (1) a significant negative correlation between relative power spectrum in $a$ with the scores in the language development domain $(r=-0.316, P=0.005)$, and (2) a significant positive correlation between $\delta / a$ ratio with the scores in language and motor domains $(r=0.305, P=0.007 ; r=$ $0.247, P=0.017$, respectively). No other significant correlations were found between the remaining quantitative EEGs variables and neurodevelopment scores.

\section{DISCUSSION/CONCLUSIONS}

Perinatal data

When birth weight $<10$ th percentile is used as the only selection criterion, "normal SGA children" may be included. In the present study, we also use maternal pregnancy information, doppler and biometrics measurements of fetal ultrasound, in addition to other neonatal measures such as head circumference or body length. According to a recent consensus, ${ }^{22}$ where these measures were considered, all our term infants SGA meet the criteria for IUGR definition. This consensus concluded that this definition (three of the following: birthweight $<10$ th percentile; head circumference $<10$ th percentile; length $<10$ th percentile; prenatal diagnosis of IUGR; and maternal pregnancy information) "could be adopted in clinical practice and in clinical trials to better focus on newborns at risk".

Visual analysis

No neonate included in this study had acute pathology or was critically ill. Thus, the purpose of the present study was to analyze specific parameters of EEG maturity and not to classify the severity of a perinatal encephalopathy. We classified the EEG background in two degrees of maturity and found that most SGA babies were in the immature group. There is a wide and rich bibliography referring to specific patterns of intra and extra-uterine EEG maturation in preterm and term "healthy" and "sick" neonates. ${ }^{23,24}$ However, to date, neither in IUGR preterm nor in SGA term babies, there are no publications on maturity parameters by visual EEG analysis of the conventional video-EEG recording. The coexistence of dysmature EEG patterns and excessive number of transients has been associated with chronic and persistent brain lesion; ${ }^{4,5}$ described in premature infants when reaching term $\mathrm{GA}^{18}{ }^{18}$ with chronic lung disease ${ }^{25}$ and with postnatal malnutrition; ${ }^{26}$ and in term neonates with other pathological intrauterine conditions. ${ }^{27}$

Asymmetry and asynchrony, together with flattening of the background EEG, most frequently unilateral, followed by a more discontinuous tracing, have been referred to as a benign variant of a more immature morphology in neonates $>35$ weeks GA. ${ }^{28}$ To date, except in the acute period of the ischemic perinatal stroke, ${ }^{21}$ asynchrony and asymmetry in term neonates have not been reported in the literature associated with other specific pathology.

Some aEEG studies conducted in neonates with IUGR have been associated with brain function immaturity. ${ }^{7,8,29}$ Our results provide new data, until now unreported, that contribute to a better knowledge on brain function maturation in these children. The analysis of the well-recognized EEG patterns of maturity in the clinical setting such as discontinuity, delta brushes, IBI duration, asymmetry, asynchrony or transients may have clinical relevance on the evaluation of SGA neonates. 
Table 3. Scores, expressed as means with their $95 \% \mathrm{Cl}$, obtained in each development domain for birthweight and for optimal cut-off points in the EEG variables of the visual analysis using Bayley-III.

\begin{tabular}{|c|c|c|c|c|c|c|}
\hline Birthweight goup & Motor & & Language & & Cognitive & \\
\hline & $94.40-104.27$ & & 88.91-101.69 & & 94.49-106.51 & \\
\hline & $104.10-114.34$ & & $100.72-116.50$ & & $105.26-117.96$ & \\
\hline \multicolumn{7}{|l|}{ Cut-off (SGA/AGA) } \\
\hline \multirow[t]{2}{*}{$\leq 11 \%(10 / 27)$} & 108.00 & & 105.37 & & 108.73 & \\
\hline & 102.83-113.17 & & $97.11-113.62$ & & $103.75-113.70$ & \\
\hline$>5 \%(28 / 7)$ & 98.61 & $(\dagger)$ & 95.60 & $(\dagger)$ & 101.61 & $(*)$ \\
\hline \multirow[t]{2}{*}{$\%$ Asymmetry } & $93.18-104.04$ & & 88.18-103.04 & & $94.60-108.62$ & \\
\hline & 109.41 & & 106.49 & & 109.55 & \\
\hline$\leq 7 \%(20 / 23)$ & $100.06-111.12$ & & $94.14-107.04$ & & $96.07-114.48$ & \\
\hline$>5$ s. $(25 / 5)$ & 98.00 & $(\dagger)$ & 93.26 & $(†)$ & 97.37 & $(\ddagger)$ \\
\hline \multirow[t]{2}{*}{ Max. IBI Duration } & $92.04-103-96$ & & $85.81-100.72$ & & 88.97-105.77 & \\
\hline & 108.52 & & 105.48 & & 111.08 & \\
\hline \multirow[t]{2}{*}{$\leq 5 \mathrm{~s} .(16 / 29)$} & $102.63-114.41$ & & $98.86-112.11$ & & $105.83-116.33$ & \\
\hline & 99.31 & $(\dagger)$ & 95.91 & $(†)$ & 99.77 & $(\ddagger)$ \\
\hline$>11 \%(26 / 5) \%$ & $92.89-105.74$ & & 88.78-103.03 & & $91.75-107.79$ & \\
\hline Delta brushes & 108.26 & & 105.83 & & 110.96 & \\
\hline$\leq 11 \%(15 / 29)$ & 102.78-113.74 & & $98.24-113.41$ & & 105.65-116.26 & \\
\hline Immature $(38 / 73)^{\mathrm{b}}$ & 100.33 & $(\dagger)$ & 97.06 & $(*)$ & 99.70 & $(\dagger)$ \\
\hline
\end{tabular}

Spectral analysis

When the sleep state has been defined by EEG and other polygraph channels, previous studies in term infants have found proportions of QS significantly reduced and increased in IS with great difficulty in exactly distinguishing sleep states in the first hours of life. ${ }^{8,19,27}$ For this, in the present work, for spectral analysis we selected EEG segments with discontinuous and/or alternating tracings regardless of behavioral state. Given the existence of asymmetry, asynchrony and transients, long epochsanalysis that included all bursts during a stable period of sleep would allow to obtain spectral values more objective than those obtained from short periods of tracing EEG arbitrarily selected.

Currently most studies aiming at analyzing neonatal brain function are performed using the quantitative analysis of EEG background. There are multiple articles on brain maturation in premature and term "healthy" and critically ill infants. ${ }^{7-11,30,31}$ However, contributions in SGA babies are more limited. In premature infants with IUGR, it has been described: (1) greater relative power in $\delta$, and lower in $\theta, a$ and $\beta$ frequency bands in relation to those non-IUGR, and (2) the relative power in the $\delta$ decreases with the increase of GA. ${ }^{12,13,29}$ In term infants, SGA neonates have been reported to have a maturational delay, defined as power spectrum increased in $\delta .^{14}$
In the present work, SGA infants, compared to AGA controls, showed higher values in the power spectrum of frequencies faster $(a$ and $\beta$ ) and lower in the $\delta$. Therefore, our findings do not agree with the maturational delay patterns described in these previous studies. This discordance could be explained by major methodological differences in the procedure and selection of the EEG recording segments for spectral analysis. In previous works in late preterm infants $^{12}$ and term neonates with 38-42 weeks of $\mathrm{GA}^{14}$ the EEG recordings were obtained in the first $24-48 \mathrm{~h}$ and in the 3-6 days of postnatal life, respectively; both studies selected short EEG segments of QS (4-10 s) that included both continuous and alternating tracings. However, the present work included term neonates a week younger that those studied by Özdemir. ${ }^{14}$ Our EEG recordings were performed between 48 and $72 \mathrm{~h}$ of life, and the EEG segments selected for the spectral analysis, exclusively with tracé alternant or discontinuous pattern, were much longer than in both previous studies.

In agreement with our results, a recent study on power spectrum analysis, conducted in the first month of postnatal life, found that those preterm infants (24-35 weeks GA) with IUGR had significantly increased relative power in $\theta, a$ and $\beta$ and reduced in $\delta$ compared to AGA peers. ${ }^{32}$ These authors, supported by published neuroimaging findings in fetuses with IUGR, ${ }^{33}$ 
suggested that SGA brain is relatively mature compared to AGA brain. However, our findings in the quantitative analysis correlate with immature patterns in visual analysis, suggesting that the reduction of relative power spectrum in $\delta$ and the increase in $a$ and $\beta$ indicate true delay in the maturation of brain function development in SGA babies.

\section{Neurodevelopment}

We found lower neurodevelopmental scores in SGA children compared to the AGA peers. Recent systematic reviews concluded that IUGR infants often display neurodevelopmental delays in early childhood ${ }^{34}$ and that late-onset IUGR babies have an increased risk of low cognitive scores. ${ }^{35}$

Our results also suggest that some patterns of EEG immaturity, such as interhemispheric asymmetry, in term SGA neonates may be warning features for development risks, as already suggested for preterm infants with IUGR. ${ }^{12}$ Due to the limited number of SGA neonates with mature EEG, we were unable to correlate neurodevelopmental scores with most of the maturity variables in this group of children. We only found a tenuous but significant association between high rates of interhemispheric asymmetry with decreased language and motor development scores. Only with this finding, where multiple variables were analyzed, a definitive conclusion cannot be obtained. However, our results, at least, invite further studies in order to assess whether the implementation of the video-EEG screening and early neurodevelopmental intervention in SGA children at risk could improve their outcome. The implementation of a video-EEG recording for all term SGA infants would have a significant increase in healthcare costs. However, any intervention capable of improving development scores even in a small quantity could have a high impact on public health. ${ }^{36}$

Although we found statistical differences in the development scores between SGA and AGA, all the children in our study, including those with EEG immature, obtained developmental scores within limits considered normal. Therefore, any neurodevelopmental intervention would be questionable. However, smallness at birth is a frequent problem. Some trials have been carried out to assess the influence of early elective delivery on neurodevelopment. ${ }^{37,38}$ None of these trials managed to find outcomes benefits in the short or long term. A systematic review concluded that "a trial designed to assess the impact of intervention in term SGA in order to improve the outcome is urgently needed" and that "an intervention capable of improving the neurodevelopmental scores from 0.3 SD to 0.15 SD would be clinically significant. ${ }^{36}$ Although, video-EEG monitoring, in itself, is not an intervention that will improve the development scores, immature EEG patterns at birth (particularly asymmetry) could potentially help identify SGA neonates at higher risk.

\section{Limitations}

IUGR is a heterogeneous condition that may have a variety of etiologic causes. Despite the prenatal and postnatal diagnostic assessment in all our SGA babies, and rigorous exclusion criteria, it is possible that some of them suffered an undiagnosed underlying etiology as genetic syndromes that influenced the results of the study.

There is a great variability in the definition of neonatal EEG variables. Except in discontinuity, the EEG variables analyzed in our study conform to the endorsed by the American Clinical Neurophysiology Society (ACNS). To consider abnormal discontinuity in an EEG tracing, most authors, including ACNS, ${ }^{23}$ accept IBI duration $>2 \mathrm{~s}$ with amplitude as low as $25 \mu \mathrm{V}$ in the tracé alternant pattern. However, when normality ranges are evaluated in the EEG maturity framework, this definition is too restrictive. Subsequently, other investigators have used an IBI amplitude of $\leq 30 \mu \mathrm{V}$ to define discontinuous pattern. ${ }^{8,11,39}$
In the present study and according with a recent guideline, ${ }^{20}$ the interrater reliability was good or excellent in the interpretation of all EEG variables of visual analysis. However, Wusthoff et al., ${ }^{40}$ using the ACNS standardized scoring system, evaluated the interrater agreement in the interpretation of neonatal EEG in hypoxic ischemic encephalopathy. The authors found very good interrater for identification of seizures and classification of EEG background, but other specific EEG features (voltage, symmetry, variability or abnormal sharp waves) showed lower interrater agreement, suggesting limited reproducibility. There are methodological differences with the present work. Wusthoff analyzed the first $3 \mathrm{~h}$ of each EEG recording performed in the first $24 \mathrm{~h}$ after birth in asphyxiated infants under hypothermia. In our work, we measured these specific EEG patterns only in the discontinuous and alternating tracings at three days of life, and some graph-elements such as transients were only counted in the interburst periods.

In the present study, there was a high percentage of children born to smoking mothers and cesarean sections. Both factors could have led to EEG modifications. However, in the multiple analysis, we did not find any level of significance between these factors with the EEG variables nor with the scores obtained in the neurodevelopment. Yerushalmy-Feler et al. ${ }^{12}$ found a significant correlation between cesarean delivery and EEG immaturity. However, apart from the methodological differences in the EEG analysis procedure, these children were premature, many of them receiving prenatal corticosteroids and the EEG recording was performed very early $(<48 \mathrm{~h}$ of life). Prenatal steroids and EEG recordings carried out before adaptation to extrauterine life can be associated with immaturity in visual and spectral EEG analysis., 8,12

Another potential limitation could be the high number (higher in the AGA group) of invalid video-EEG recordings and of children lost in the follow-up and/or without collaboration in their neurodevelopment assessment. However, it is easy to understand that there were important practical barriers for the recruitment of healthy neonates (AGA group) for intensive videoEEG monitoring $(>3 \mathrm{~h})$, and undergo another new EEG recording when there was a previous invalid EEG tracing. Following the recommendations of the Spanish Society of Neonatology, from birth, SGA babies are periodically monitored at neonatal followup hospital visits. This would explain that most parents of SGA children were aware of the possible postnatal and developmental risks of their children, and of their greater compliance with this research project.

Using the ROC curves and the Youden Index, we obtained cutoff points as markers of EEG maturity to discriminate between SGA and AGA neonates. These cut-off points were also used later to evaluate their involvement in neurodevelopment. Compared with AGA, SGA babies had both: (1) higher values than these cut-off points, and (2) lower developmental scores. Therefore, there may be some bias when lower neurological development outcomes are obtained in children in whom only higher rates of EEG immaturity are considered. However, taking into account only the group of SGA infants, we found a significant correlation between interhemispheric asymmetry with lower scores in motor and language development.

\section{Conclusions}

Using conventional video-EEG recordings in the third day of extrauterine life, the present prospective study provides specific findings of EEG immaturity in SGA children so far not reported: (1) In the visual analysis of the EEG background, it is common to find high rates of specific EEG patterns of immaturity and excessive transients per hour, and (2) in the quantitative analysis of alternating or discontinuous EEG tracings, higher values in the relative power spectrum to faster frequencies $(a$ and $\beta$ ) and lower in delta can be found. 
In the neurodevelopmental assessment, compared to AGA, SGA children can obtain lower scores in motor, language and cognitive development. We also provide relevant findings in the clinical setting, to date not referenced in the literature. Term neonates (AGA and SGA) with excessive IBI duration and high rates of immature EEG patterns, such as discontinuity, interhemispheric asymmetry of the bursts or percentage of bursts with delta brushes in their video-EEG recordings at third day after birth, may have unfavorable prognostic implications resulting in decreased scores in the neurodevelopmental assessment in early childhood. However, further studies including a greater number of SGA infants are needed in order to determine what rates of immaturity EEG could predict outcomes in low-birth-weight term neonates.

\section{ACKNOWLEDGEMENTS}

Sebastian McLean was responsible for the English version of the manuscript. Several phases of this study were supported by Caixabank Social Work Foundation: (1) Acquisition of Insight II software, and (2) Hiring an experienced psychologist in psychomotricity and statistical data management. Reference number of the research project of the Universidad de La Laguna: SALUTRA05.

\section{AUTHOR CONTRIBUTIONS}

Dr. Castro Conde conceptualized and designed the study, drafted the initial manuscript, and reviewed and revised the manuscript. Drs. González Campo, Quintero Fuentes and Jiménez Sosa designed the data collection instruments, coordinated and supervised data collection, and reviewed and revised the manuscript. Drs. González González, González Barrios and Reyes Millán carried out the initial analyses, and critically reviewed the manuscript for important intellectual content. All authors approved the final manuscript as submitted and agree to be accountable for all aspects of the work.

\section{ADDITIONAL INFORMATION}

The online version of this article (https://doi.org/10.1038/s41390-019-0693-0) contains supplementary material, which is available to authorized users.

Competing interests: The authors have no conflicts of interest to declare. There are no prior publications or submissions with any overlapping information, including study data and patients in an abstract or poster. McLean Language School (MLS) provides a translation, editing and review service to papers to be published by staff members of the Universidad de La Laguna (ULL). MLS declares no competing interest in relation to this article.

Statement of ethics: The study was approved by the Research Ethics Committee of our Hospital. Written informed parental consent was obtained after delivery.

Publisher's note Springer Nature remains neutral with regard to jurisdictional claims in published maps and institutional affiliations.

\section{REFERENCES}

1. Bassan, H. et al. Intrauterine growth-restricted neonates born at term or preterm: how different? Pediatr. Neurol. 44, 122-130 (2011).

2. Sanz-Cortes, M. et al. Association of brain metabolism with sulcation and corpus callosum development assessed by MRI in late-onset small fetuses. Am. J. Obstet. Gynecol. 212, e1-e8 (2015).

3. Eixarch, E., Muñoz-Moreno, E., Bargallo, N., Batalle, D. \& Gratacos, E. Motor and cortico-striatal-thalamic connectivity alterations in intrauterine growth restriction. Am. J. Obstet. Gynecol. 214, e1-e9 (2016).

4. Hayakawa, F., Okumura, A., Kato, T., Kuno, K. \& Watanabe, K. Dysmature EEG pattern in EEGs of preterm infants with cognitive impairment: maturation arrest caused by prolonged mild CNS depression. Brain Dev. 19, 122-125 (1997).

5. Watanabe, K., Hayakawa, F. \& Okumura, A. Neonatal EEG: a powerful tool in the assessment of brain damage in preterm infants. Brain Dev. 21, 361-367 (1999).

6. Biagioni, E. et al. Maturation of cerebral electrical activity and development of cortical folding in young very preterm infants. Clin. Neurophysiol. 118, 53-59 (2007).

7. O'Toole, J. M., Pavlidis, E., Korotchikova, I., Boylan, G. B. \& Stevenson, N. J. Temporal evolution of quantitative EEG within 3 days of birth in early preterm infants. Sci. Rep. 9, 4859 (2019).
8. Castro Conde, J. R. et al. Visual and quantitative EEG analysis in healthy term neonates within the first $6 \mathrm{~h}$ and the third day of birth. Pediatr. Neurol. 77, 54-60 (2017).

9. O'Toole, J. M., Boylan, G. B., Vanhatalo, S. \& Stevenson, N. J. Estimating functional brain maturity in very and extremely preterm neonates using automated analysis of the electroencephalogram. Clin. Neurophysiol. 127, 2910-18. (2016).

10. Niemarkt, H. J. et al. Maturational changes in automated EEG spectral power analysis in preterm infants. Pediatr. Res. 70, 529-534 (2011).

11. Victor, S., Appleton, R. E., Beirne, M., Marson, A. G. \& Weindling, A. M. Spectral analysis of electroencephalography in premature newborn infants: normal ranges. Pediatr. Res. 57, 336-341 (2005).

12. Yerushalmy-Feler, A. et al. Electroencephalographic characteristics in preterm infants born with intrauterine growth restriction. J. Pediatr. 164, 756-61. (2014).

13. Schwindt, E. et al. Being born small for gestational age influences amplitudeintegrated electroencephalography and later outcome in preterm infants. Neonatology 108, 81-87 (2015).

14. Özdemir, O. M., Ergin, H. \& Sahiner, T. Electrophysiological assessment of the brain function in term SGA infants. Brain Res. 1270, 33-38 (2009).

15. González González, N. L. et al. Customized weight curves for Spanish fetuses and newborns. J. Matern. Fetal Neonatal Med. 27, 1495-1499 (2014).

16. Sociedad Española de Ginecología y Obstetricia [homepage on the internet]. Documentos de Consenso S.E.G.O. 187 Group. Defectos del crecimiento fetal (update 2015). https://sego.es/Medicina_Perinatal\#docsconsensopub.

17. Amiel-Tison, C. Update of the Amiel-Tison neurologic assessment for the term neonate or at 40 weeks corrected age. Pediatr. Neurol. 27, 196-212 (2002).

18. Castro Conde, J. R. et al. Influence of extrauterine life on ontogenic EEG parameters in preterm newborns with and without major ultrasound brain lesions. Clin. Neurophysiol. 116, 2796-2809 (2005).

19. Curzi-Dascalova, L., Peirano, P. \& Morel-Kahn, F. Development of sleep states in normal premature and full-term newborns. Dev. Psychobiol. 21, 431-444 (1988).

20. Koo, T. K. \& Li, M. Y. A guideline of selecting and reporting intraclass correlation coefficients for reliability research. J. Chiropr. Med. 15, 155-163 (2016).

21. Castro Conde, J. R. et al. EEG findings and outcomes of continuous video-EEG monitoring started prior to initiation of seizure treatment in the perinatal stroke. Early Hum. Dev. 120, 1-9 (2018).

22. Beune, I. M. et al. Consensus based definition of growth restriction in the newborn. J. Pediatr. 196, 71-76 (2018).

23. Lamblin, M. D. et al. Electroencephalography of the premature and term newborn. maturational aspects and glossary. Neurophysiol. Clin. 29, 123-219 (1999).

24. Tsuchida, T. N. et al. American Clinical Neurophysiology Society standardized EEG terminology and categorization for the description of continuous EEG monitoring in neonates: report of the American Clinical Neurophysiology Society Critical Care Monitoring Committee. J. Clin. Neurophysiol. 30, 161-173 (2013).

25. Hahn, J. S. \& Tharp, B. R. The dysmature EEG pattern in infants with bronchopulmonary dysplasia and its prognostic implications. Electroencephalogr. Clin. Neurophysiol. 76, 106-13. (1990).

26. Okumura, A. et al. Nutritional state, maturational delay on electroencephalogram, and developmental outcome in extremely low birth weight infants. Brain Dev. 32, 613-618 (2010).

27. Castro Conde, J. R. et al. Video-EEG recordings in full-term neonates of diabetic mothers: observational study. Arch. Dis. Child Fetal Neonatal Ed. 98, F493-F498 (2013).

28. O'Brien, M. J., Lems, Y. L. \& Prechtl, H. F. Transient flattenings in the EEG of newborns-a benign variation. Electroencephalogr. Clin. Neurophysiol. 67, 16-26 (1987).

29. Benavente-Fernández, I. et al. Amplitude-integrated EEG and brain sparing in preterm small-for-gestational-age infants. J. Clin. Neurophysiol. 34, 456-60. (2017).

30. Schumacher, E. M. et al. Automated spectral EEG analyses of premature infants during the first three days of life correlated with developmental outcomes at 24 months. Neonatology 103, 205-212 (2013).

31. Shellhaas, R. A. et al. Neonatal sleep-wake analyses predict 18 -month neurodevelopmental outcomes. Sleep 40, https://doi.org/10.1093/sleep/zsx144 (2017).

32. Cohen, E. et al. EEG power spectrum maturation in preterm fetal growth restricted infants. Brain Res. 1678, 180-186 (2018).

33. Businelli, C., de Wit, C., Visser, G. H. \& Pistorius, L. R. Ultrasound evaluation of cortical brain development in fetuses with intrauterine growth restriction. J. Matern. Fetal Neonatal Med. 10, 1-6 (2014).

34. Levine, T. A. et al. Early childhood neurodevelopment after intrauterine growth restriction: a systematic review. Pediatrics 135, 26-141. (2015).

35. Figueras, F. et al. Neurobehavior in term, small-for-gestational age infants with normal placental function. Pediatrics 124, e934-e941 (2009). 
Assessment of neonatal EEG background and neurodevelopment in full-term... JR Castro Conde et al.

36. Arcangeli, T., Thilaganathan, B., Hooper, R., Khan, K. S. \& Bhide, A. Neurodevelopmental delay in small babies at term: a systematic review. Ultrasound Obstet Gynecol. 40, 267-275 (2012).

37. van Wik, L. et al. Effects on (neuro)developmental and behavioral outcome at 2 years of age of induced labor compared with expectant management in intrauterine growth-restricted infants: long-term outcomes of the DIGITAT trial. Am. J. Obstet. Gynecol. 206, e1-e7 (2012).

38. Walker, D. M. et al. The Growth Restriction Intervention Trial: long-term outcomes in a randomized trial of timing of delivery in fetal growth restriction. Am. J. Obstet. Gynecol. 204, e1-e9 (2011).

39. Biagioni, E. et al. Background EEG activity in preterm infants: correlation of outcome with selected maturational features. Electroencephalogr. Clin. Neurophysiol. 91, 154-162 (1994)

40. Wusthoff, C. J. et al. Interrater agreement in the interpretation of neonatal electroencephalography in hypoxic-ischemic encephalopathy. Epilepsia 58, 429-35 (2017) c) (1) $\Theta$ Open Access This article is licensed under a Creative Commons Attribution-NonCommercial-NoDerivatives 4.0 International License, which permits any non-commercial use, sharing, distribution and reproduction in any medium or format, as long as you give appropriate credit to the original author(s) and the source, and provide a link to the Creative Commons license. You do not have permission under this license to share adapted material derived from this article or parts of it. The images or other third party material in this article are included in the article's Creative Commons license, unless indicated otherwise in a credit line to the material. If material is not included in the article's Creative Commons license and your intended use is not permitted by statutory regulation or exceeds the permitted use, you will need to obtain permission directly from the copyright holder. To view a copy of this license, visit http://creativecommons.org/licenses/by-nc-nd/4.0/.

(c) The Author(s) 2019 